\title{
Toxicity screening of two prevalent metal organic frameworks for therapeutic use in human lung epithelial cells
}

This article was published in the following Dove Press journal: International Journal of Nanomedicine

\author{
Alixandra Wagner ${ }^{1 * *}$ \\ Qian Liu',* \\ Olivia L Rose ${ }^{1, *}$ \\ Anna Eden' \\ Aishwarya Vijay' \\ Yon Rojanasakul ${ }^{2}$ \\ Cerasela Zoica Dinu'
}

'Department of Chemical and Biomedical Engineering, Benjamin M. Statler College of Engineering and Mineral Resources, West Virginia University, Morgantown, WV 26506, USA; ${ }^{2}$ Department of Basic Pharmaceutical Sciences, West Virginia University, Morgantown WV 26506, USA

*These authors contributed equally to this work
Correspondence: Cerasela Zoica Dinu Department of Chemical and Biomedical Engineering, M. Statler College of Engineering and Mineral Resources, West Virginia University, Benjamin, PO Box 6102, Morgantown, WV 26506, USA

Tel +I 3042939338

Fax +I 3042934139

Email cerasela-zoica.dinu@mail.wvu.edu
Introduction: The flexibility and tunability of metal organic frameworks (MOFs), crystalline porous materials composed of a network of metal ions coordinated by organic ligands, confer their variety of applications as drug delivery systems or as sensing and imaging agents. However, such properties also add to the difficulty in ensuring their safe implementation when interaction with biological systems is considered.

Methods: In the current study, we used real-time sensorial strategies and cellular-based approaches to allow for fast and effective screening of two MOFs of prevalent use, namely, MIL-160 representative of a hydrophilic and ZIF- 8 representative of a hydrophobic framework. The two MOFs were synthesized "in house" and exposed to human bronchial epithelial (BEAS-2B) cells, a pertinent toxicological screening model.

Results: Analysis allowed evaluation and differentiation of particle-induced cellular effects as well identification of different degrees and routes of toxicity, all in a high-throughput manner. Our results show the importance of performing screening toxicity assessments before introducing MOFs to biomedical applications.

Discussion: Our proposed screening assays could be extended to a wider variety of cell lines to allow for identification of any deleterious effects of MOFs, with the range of toxic mechanisms to be differentiated based on cell viability, morphology and cell-substrate interactions, respectively.

Conclusion: Our analysis highlights the importance of considering the physicochemical properties of MOFs when recommending a MOF-based therapeutic option or MOFs implementation in biomedical applications.

Keywords: metal organic frameworks, real-time assay, high-throughput assessment

\section{Introduction}

Metal organic frameworks (MOFs) are crystalline porous materials with ultrahigh surface areas, ${ }^{1-3}$ consisting of metal ions or clusters linked with organic ligands via coordination bonds. ${ }^{4-6}$ The large number of combinations of metal ions and organic ligands available during such material synthesis confers a high degree of tunability of MOF's geometry, size, porosity and functionality, respectively. ${ }^{5,6}$ Such features conversely elicit MOF integration in numerous applications ranging from gas capture and storage , $^{7,8}$ to water adsorption and from catalysis to electrical-based applications. $^{9-12}$

Recently, MOFs have also gained interest and use in biomedical applications, ${ }^{13}$ both for the formation of contrast agents for MRI enhancement ${ }^{13,14}$ and as agents for drug 
delivery strategies. ${ }^{13}$ The ability to tune their structures as well as their porosity to target specific areas of the body has allowed for adequate adsorption of selective drugs or molecules as well as user-directed delivery. ${ }^{15} \mathrm{MOF}$ integration also led to better contrast and longer duration of imaging when used for MRI, all relative to traditional contrast agents ${ }^{14,16,17}$ or time-controlled delivery of covalently or noncovalently bound drugs to selected diseased tissue, all while preventing side effects of the surrounding healthy ones. ${ }^{18-20}$

However, recent concerns over MOF or MOF-related product safety have been raised. Preliminary toxicological assessment studies showed that the presence of metal atoms in MOFs coordinated bonds structure could create a local imbalance in the exposed biological system to affect homeostasis, both extracellularly and intracellularly, ${ }^{19}$ with the degree of the homeostatic changes being dependent on material's composition and structure. Studies by Tamames-Tabar et al., showed, for instance, that the toxicity of fourteen different MOFs containing varying metal ions (e.g., iron, zinc, and zirconium) and organic linkers (e.g., carboxylates or imidazolates) on human cervical carcinoma epithelial cells (HeLa) and in a murine macrophage cell line (J774) was dependent on the composition of the individual MOF being tested as well as the cell line being assessed. ${ }^{21}$ Analysis also showed that iron-based MOFs had lower toxicity relative to both zirconium- and zinc-MOFs, with $\mathrm{J} 774$ cells experiencing a greater toxicity due to the faster internalization of the tested material. ${ }^{21}$ Complementary work by Ruyra et al., evaluated and categorized toxicity of sixteen uncoated MOFs in exposed zebrafish embryos, with results sorting deleterious effects based on material structure and the individual-related leaching of metal ions. ${ }^{22}$ Additionally, Ren et al., found that IRMOF-3, a rigid cubic zinc-based MOF, had a time- and concentration-dependent toxicity on rat pheochromocytoma cells starting at concentrations as low as $100 \mu \mathrm{g} / \mathrm{mL},{ }^{23}$ while Grall et al., observed low toxicity of mesoporous metals-based (e.g., iron, aluminum, chromium) MOFs when exposed to two hepatic cell lines (HepG2 and Hep3B) and two lung cell lines (A549 and Calu-3 respectively) at doses ranging from 10 to $100 \mu \mathrm{g} /$ $\mathrm{mL} .{ }^{24}$

However, while highly comprehensive, these available studies are mostly based on single-point assays (e.g., 4 succinate dehydrogenase activity - MTT - assay) that do not allow real-time assessment of toxicity nor provide insights into time-dependent windows of exposure. Considering that such single-point assays also suffer from interference from reagents normally used in classical cellular approaches that could potentially influence MOFinduced toxicity assessment, ${ }^{25}$ we proposed to screen for cellular behavior upon exposure to MOFs of prevalent use by using only cell-based bio-sensorial approaches. To demonstrate the validity and applicability of the proposed approaches, we used MIL-160, a microporous hydrophilic aluminum-based $\mathrm{MOF}^{10,26}$ with a five-membered ring and an oxygen heteroatom, ${ }^{10}$ and ZIF-8, a hydrophobic framework of zeolitic imidazolate. ${ }^{10,27}$ Human bronchial epithelial (BEAS-2B) cells were employed as a pertinent model for toxicity assessment. ${ }^{28}$ MOF selection was based on their demonstrated wide integration for gas storage applications, ${ }^{29}$ enzyme-based green technologies ${ }^{10}$ and drug delivery systems, ${ }^{30,31}$ while cell selection was based on demonstrated intrinsic sensitivity of such systems to convert biological activity into electrical measurements to be recorded in real time with an electric cell impedance sensing platform (ECIS). ${ }^{25}$ By simply relying on the natural sensitivity of cells, our analysis demonstrates highthroughput, rapid and accurate screening and differentiation of toxicity based on MOFs physicochemical properties, as well as the capability for extension and large-scale screening of other MOF materials.

\section{Materials and methods Preparation of MIL-160}

MIL-160 was synthesized by linking aluminum chloride hexahydrate (99\%, Acros Organics) with 2, 5-furandicarboxylic acid (98\%, Alfa Aesar), through a hydrothermal method. ${ }^{32}$ Specifically, a precursor compromised of dissolved 2, 5-furandicarboxylic acid, aluminum chloride hexahydrate and sodium hydroxide (extra pure, Acros Organics) was suspended in deionized water in a mole ratio of 1.0: 1.0: 2.1: 1000. The precursor was subsequently dosed in a Teflonlined stainless steel autoclave and heated at a temperature of $363 \mathrm{~K}$ for $24 \mathrm{hrs}$. After hydrothermal reaction and cooling to room temperature, the products were filtered on cellulose filters of grade 1 (Whatman) and washed extensively with deionized water to remove any unreacted species. The filter paper was then dried overnight at room temperature and the resulting products were removed and stored in a vacuum chamber, again, at room temperature.

\section{Preparation of ZIF-8}

ZIF-8 was synthesized by rapidly mixing zinc nitrate (98\%, Acros Organics) and 2-methylimidazole (97\%, Alfa Aesar) 
solutions, at room temperature, for about 10 mins. $^{33}$ Initially, solutions A and B were prepared, with solution A being prepared by dissolving $0.2 \mathrm{~g}$ zinc nitrate hexahydrate in $5 \mathrm{~mL}$ deionized water and solution $\mathrm{B}$ by dissolving $2.0 \mathrm{~g}$ 2-methylimidazole also in $5 \mathrm{~mL}$ deionized water. The solutions were then mixed under uniform mechanical stirring and at room temperature. The resulting product was collected by centrifugation at $5000 \mathrm{rpm}$ for $8 \mathrm{mins}$, washed with deionized water several times to remove any nonprecipitated species, dried at room temperature and subsequently stored in a vacuum chamber, again at room temperature.

\section{Material characterization}

Surface morphology and elemental composition of MIL-160 and ZIF-8 were investigated using Hitachi S-4700 Field Emission Scanning Electron Microscope (Hitachi High-Technologies Corporation) equipped with energy dispersive X-ray (EDX) spectroscopy. For these analyses, dry powders of the samples were mounted onto carbon tape and investigated using an accelerating voltage of $5.0 \mathrm{kV}$.

Crystalline phases of the synthesized MIL-160 and ZIF-8 MOFs were confirmed by powder x-ray diffraction (PXRD) analysis with experiments performed at room temperature under ambient conditions on PANalytical $X$ 'Pert Pro X-ray Diffractometer with $\mathrm{CuKa}$ radiation at $40 \mathrm{kV}$ and $40 \mathrm{~mA}$, respectively.

\section{Cell culture}

Immortalized human bronchial epithelial (BEAS-2B, American Type Culture Collection - ATCC) cells were cultured in Dulbecco's Modified Eagle Medium (DMEM; Corning) media containing 5\% FBS, 1\% L-glutamine and $1 \%$ penicillin-streptomycin (reagents purchased from Life Technologies). The cells were passaged regularly using $0.25 \%$ trypsin (Invitrogen) and incubated at $37^{\circ} \mathrm{C}, 5 \%$ $\mathrm{CO}_{2}$ and $80 \%$ relative humidity. Before each experiment, cells were grown to a confluent monolayer. Control experiments with human non-small cancer lung (H460) cells purchased from ATCC were also performed. For these, H460 cells were cultured in Roswell Park Memorial Institute-1640 medium (RPMI, Gibco) supplemented with $10 \%$ FBS, $1 \%$ L-glutamine and 1\% penicillin/streptomycin. Cells were passaged regularly using $0.25 \%$ trypsin and maintained in a humidified atmosphere at $37^{\circ} \mathrm{C}$ and $5 \% \mathrm{CO}_{2}$.

\section{Dose-response curves}

BEAS-2B and H460 cells were seeded in 12-well plates (Falcon) at a density of $2.0 \times 10^{5}$ cells $/ \mathrm{mL}$. After $24 \mathrm{hrs}$, the cells were exposed to MIL-160 or ZIF-8 at the doses of 1, $10,50,100,250,500$ and $750 \mu \mathrm{g} / \mathrm{mL}$, respectively, with such doses formed by serial dilutions from original stock concentrations in their respective cells' media. Before any of the intended cellular exposures, the samples were sonicated for 8-10 mins using of a bath sonicator (Branson). After $24 \mathrm{hrs}$ of exposure, the cells were trypsinized, stained with $0.4 \%$ trypan blue solution (Invitrogen) and $10 \mu \mathrm{L}$ of such stained cells was subsequently added to a hemocytometer (Hausser Scientific). The number of cells in the four outer grids of the hemocytometer were counted using a Leica DM IL optical microscope (Leica Microsystems) and a $10 \times$ objective to determine the $\mathrm{IC}_{50}$ value, ie, the half maximum inhibitory concentration.

\section{ECIS}

Real-time analysis of BEAS-2B cells behavior was mon-

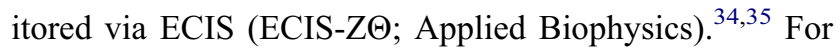
such analyses, 96-well plates (96W1E+ or 96W20idf) were used; the electrodes were first stabilized for $2 \mathrm{hrs}$ with $200 \mu \mathrm{L}$ of DMEM to help minimize any potential drift during the experiment. BEAS-2B cells were subsequently seeded at a density of $2.0 \times 10^{5}$ cells $/ \mathrm{mL}$ and allowed to form a confluent monolayer for $24 \mathrm{hrs}$. After $24 \mathrm{hrs}$, the cells were exposed to suspensions of MIL-160 or ZIF-8 dispersed in media (as previously described) at doses below, at and above their determined $\mathrm{IC}_{50}$ values (see section above). Cellular behavior was monitored in real-time over $72 \mathrm{hrs}$ of exposure with changes in cellular resistance and alpha's parameter (detailing the changes in the current between the ventral surface of the cell and electrode) $)^{36}$ being continuously monitored during this time. Media with MIL-160 or media with ZIF-8, at each exposure dose, served as blanks while cells only in media served as controls.

\section{Statistical analyses}

All graphs are presented as the mean value of the number of indicated replicates with $( \pm)$ SE bars. GraphPad Prism (GraphPad Software) was used for the determination of the $\mathrm{IC}_{50}$ values, with a sigmoidal dose response (four-parameter dose-response curve) being chosen for the fit. 


\section{Results and discussion}

We implemented a screening strategy based on ECIS ${ }^{34,35}$ for a common in vitro model cell line ${ }^{28}$ (BEAS-2B) to assess the safety of MOFs of prevalent use in real-time and in a high-throughput fashion. For the assessment, MOFs were differentiated based on their physicochemical characteristics since previous studies showed that materials' properties determine the overall toxicity in an exposed biological system. Specifically, the chosen MOFs differed in their surface morphology, with MIL-160 displaying a cube-like morphology with distinct edges and points (Figure 1A) and ZIF-8 displaying a flower-like morphology with softer edges (Figure 1B) as shown by scanning electron microscopy (SEM) analysis. Both MOFs displayed regular sizes of $8-10 \mu \mathrm{m}$ for MIL-160 and 1-2 $\mu \mathrm{m}$ for ZIF-8, respectively, with the observed differences being based on their overall aspect ratios.

Elemental composition of the two MOFs was determined by energy dispersive X-ray (EDX) spectroscopy and showed that MIL-160 composition consisted mainly of carbon and oxygen resulting from the integration of the 2, 5-furandicarboxylic acid linker, as well as aluminum from the presence of aluminum chloride hexahydrate metal clusters (Figure 1C). The elemental composition of ZIF-8 consisted of carbon, nitrogen and zinc, all consistent with the make-up of its imidazolate linker and metal ion, zinc, respectively.
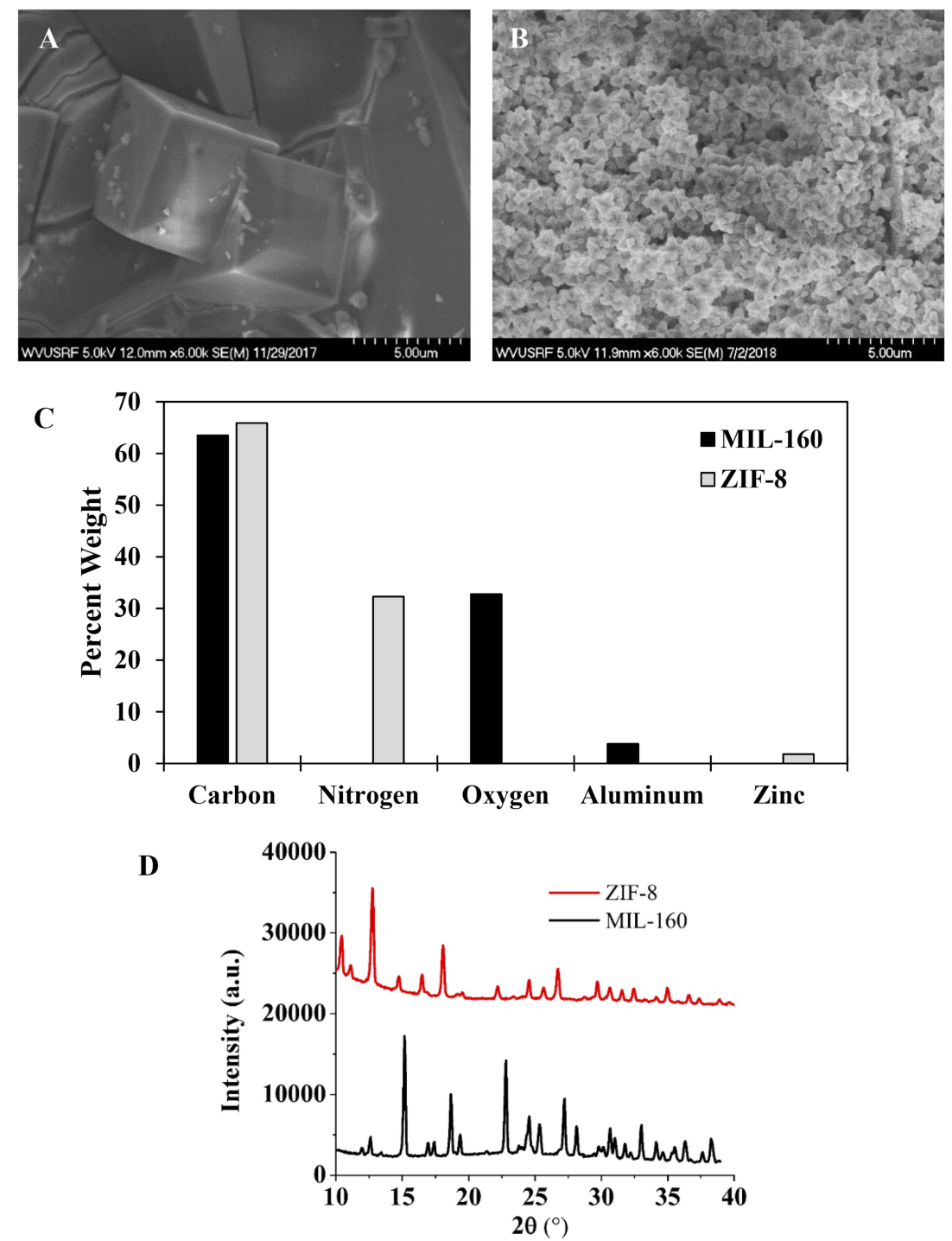

Figure I Surface morphology of (A) MIL-I60 and (B) ZIF-8 as determined by SEM. (C) Elemental composition of MIL-I60 and ZIF-8 as determined by EDX. (D) Crystalline structures of the two MOFs of prevalent use as determined by PXRD.

Abbreviations: SEM, scanning electron microscopy; EDX, energy dispersive X-ray spectroscopy; MOFs, metal organic frameworks; PXRD, powder X-ray diffraction. 
Powder x-ray diffraction (PXRD) analysis confirmed both MIL-160 and ZIF-8 crystalline phases (Figure 1D) with characteristic peaks of MIL- $160^{11}$ at $15.2^{\circ}, 18.8^{\circ}$, $22.8^{\circ}$ and $27^{\circ}$ as assigned to the planes (031), (022), (051) and (502) respectively. Complementary, characteristic peaks of ZIF-8 at $10.33^{\circ}, 12.8^{\circ}, 14.7^{\circ}, 16.5^{\circ}$ and $18^{\circ}$ were attributed to planes (002), (112), (022), (013) and (222) respectively, consistent with previous studies. 33,37

For the proposed toxicity screening of the two MOFs, a dose-response curve was first established. Human bronchial epithelial (BEAS-2B) and human non-small cancer lung (H460) cells were used. Specifically, cells were exposed to either MIL-160 or ZIF-8 MOFs at doses of 1 , $10,50,100,250,500$ and $750 \mu \mathrm{g} / \mathrm{mL}$; the range of doses was chosen to represent the potential exposures used when biological applications are considered. ${ }^{38}$ The doseresponse curves of BEAS-2B cells exposed to MIL-160 and ZIF-8 are displayed in Figure 2A and B, respectively. The analysis of the $\mathrm{IC}_{50}$ values (i.e., concentration of MOF required to inhibit cell growth by $50 \%$ ) was determined from these curves, with exposure to MIL-160 and ZIF-8 eliciting $\mathrm{IC}_{50}$ values of 421 and $57 \mu \mathrm{g} / \mathrm{mL}$, respectively, and indicating a much lower degree of toxicity for MIL160 relative to the ZIF-8 particles. The dose-response curves for H460 cells are displayed in Figure 2C and D,

A

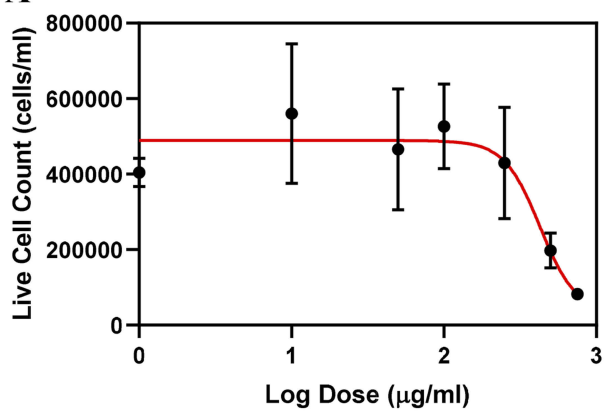

C

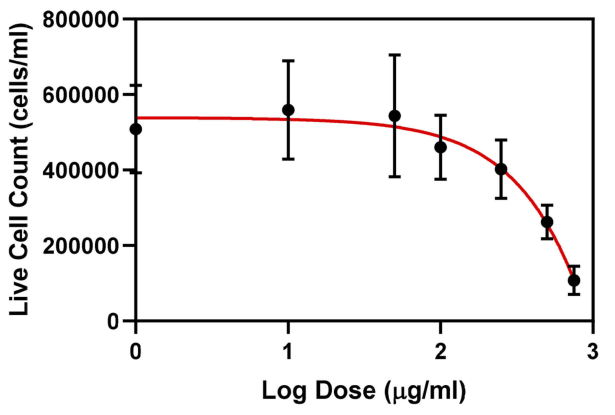

respectively. The analysis of $\mathrm{IC}_{50}$ values was determined from these curves, with exposure to MIL-160 and ZIF-8 eliciting $\mathrm{IC}_{50}$ values of $433 \mu \mathrm{g} / \mathrm{mL}$ and $61 \mu \mathrm{g} / \mathrm{mL}$, respectively, values which were similar to the ones obtained for the BEAS-2B. Because BEAS-2B cells were previously established as an acceptable model cell line in a variety of toxicity studies, ${ }^{28}$ we decided to focus the remaining analysis solely on BEAS-2B cells.

The observed differences in toxicity between the two prevalent MOFs are presumably due to their different characteristics, one of which is hydrophobicity. This is supported by a previous analysis that showed that hydrophobicity plays critical roles in nanomaterial interactions with biological systems, with protein absorption and corona formation at their interfaces to influence materialcellular uptake ${ }^{39}$ and with hydrophobic nanoparticles known to be more disruptive to cellular membranes because of theirability to localize within to thus remove lipids from their structural bilayer. ${ }^{39}$ Specifically, Tamames-Tabar et al., found a correlation between MOF degree of hydrophobicity and toxicity. ${ }^{21}$ Analysis showed that as the $\log P$ for the MOF increased, the degree of toxicity generally increased, indicating a higher degree of toxicity for hydrophobic MOFs, ${ }^{21}$ consistent with the results of this study. Numerous other studies have also found that an increase

B

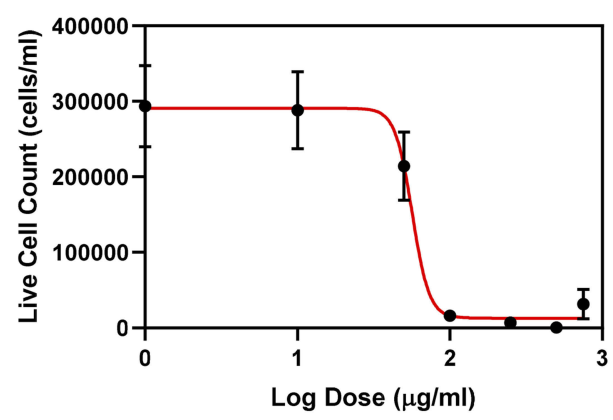

D

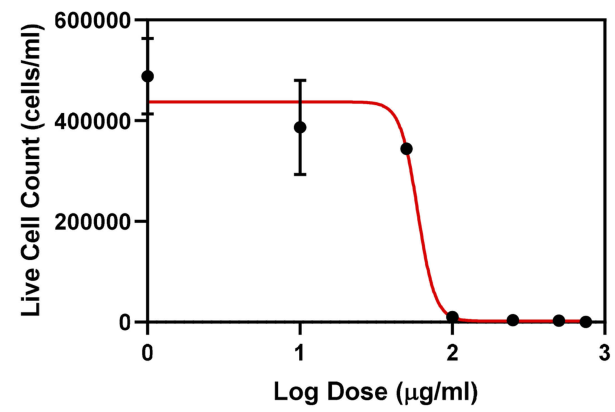

Figure 2 Dose-response curves ( \pm SE bars) of BEAS-2B cells exposed to (A) MIL-I60 and (B) ZIF-8 ( $\geq 4)$ and H460 cells exposed to MIL-I60 ( $n=6)(\mathbf{C})$ and $Z$ IF-8 ( $=2)(\mathbf{D})$ respectively. 
in hydrophobicity caused an increased toxicity of nanomaterials. ${ }^{40,41}$ For example, Farcal et al., showed that toxicity increased when $\mathrm{TiO}_{2}$ nanomaterials were coated with a hydrophobic coating, ${ }^{40}$ presumably due to interactions of such coating with the lipid layers of the cell's membrane, ${ }^{39,40}$ with such interactions resulting in changes to membrane integrity, cell signaling, ${ }^{42}$ as well as increased uptake. $^{43}$ Additionally, in regard to the uptake of such nanomaterials, previous studies have found that an increase in uptake results in an increase in cellular toxicity presumably due to greater effects on the metabolic pathways of the exposed cells. $^{21,44}$

In addition to the differences in hydrophobicity, the elemental composition, morphology and size of MIL-160 and ZIF-8 could likely influence their degree of toxicity. Specifically, zinc, the constituting metal for ZIF-8 was shown to have a higher degree of toxicity relative to other metals (e.g., iron or zirconium), with the toxicity being due to its ability to modify cellular metabolism to ultimately lead to cellular damage. ${ }^{21}$ In addition, ZIF-8 displayed a smaller particle size relative to MIL-160; smaller particles were previously shown to result in higher surface area for internal cellular protein binding ${ }^{45}$ along with higher degrees of toxicity. ${ }^{46}$ MIL-160 displayed a morphology with sharper edges relative to ZIF-8 which has the potential to disturb cellular membranes ${ }^{47}$ however to a smaller degree .

Real-time screening using Electric Cell Impedance Sensing (ECIS) platform ${ }^{48}$ was performed at doses below, at and above each MOF's respective $\mathrm{IC}_{50}$ value $(200,400$ and $800 \mu \mathrm{g} / \mathrm{mL}$ for MIL-160 and 20, 60 and $100 \mu \mathrm{g} / \mathrm{mL}$ for ZIF8 ), with exposure monitored continuously over a $72 \mathrm{hrs}$ period. ECIS was previously shown to provide a mean to assess cell-cell and cell-substrate interactions, cell morphology, motility, and coverage at a nanoscale resolution and in a high-throughput manner. ${ }^{34,35,49}$ Representative analysis of cellular resistance monitored using the ECIS system provided an indication of cell coverage, morphology and viability respectively. ${ }^{34,35}$

The resistance trends displayed by the BEAS-2B cells over the $72 \mathrm{hrs}$ of exposure showed to be dependent on the MOF physicochemical characteristics and exposure doses being used. Specifically, cells exposed to MIL-160 displayed a dose-dependent decrease in their resistance relative to control cells (Figure 3A), with the cells exposed to $800 \mu \mathrm{g} / \mathrm{mL}$ displaying a complete loss in resistance within $20 \mathrm{hrs}$ of exposure, thus indicating a loss in the cell-cell interaction and monolayer formation, as well as changes in cellular viability. ${ }^{48}$ Consistent with the dose-response curves and the $\mathrm{IC}_{50}$ value, cells exposed to MIL-160 at $400 \mu \mathrm{g} / \mathrm{mL}$ displayed a resistance that was approximately half of that of the control (cells that were not exposed). This result persisted over the whole 72 hrs of observation. Complementary cells exposed to $200 \mu \mathrm{g} / \mathrm{mL}$ displayed slightly lower resistances relative to control cells, with such results being recorded over the full exposure time. Unlike the cells exposed to MIL-160, cells exposed to ZIF-8 at doses below $(20 \mu \mathrm{g} / \mathrm{mL})$ and at the material's
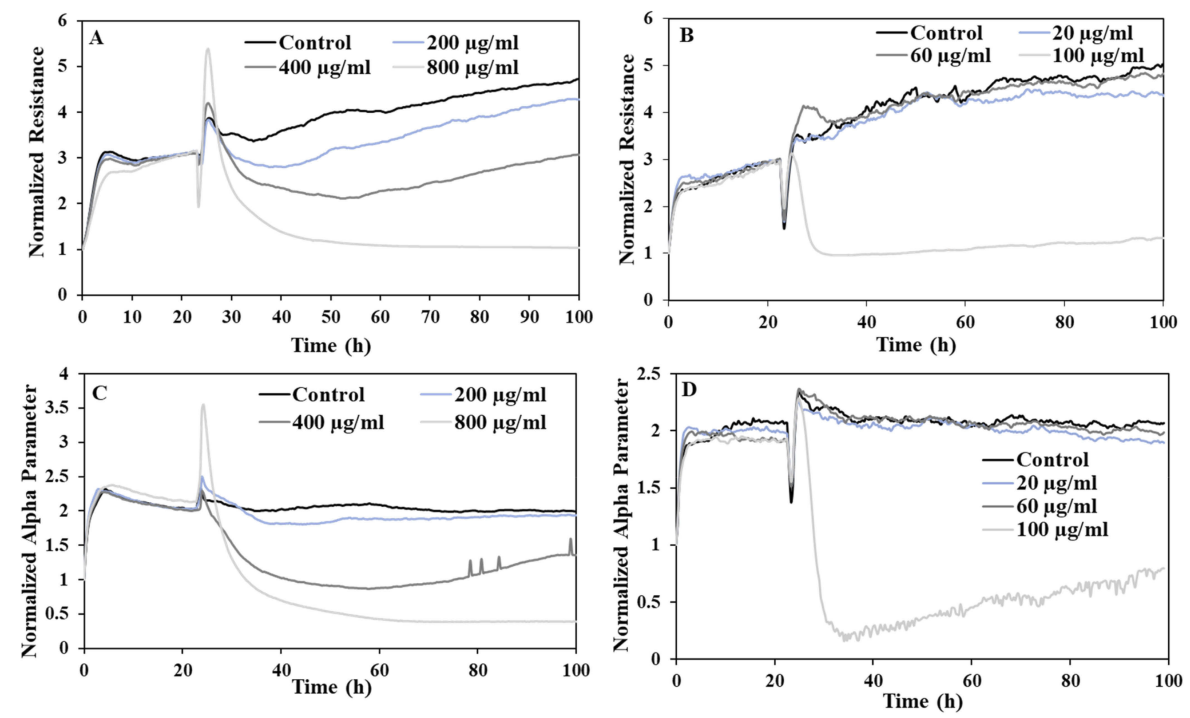

Figure 3 Representative normalized resistance of BEAS-2B cells after exposure to (A) MIL-I60 and (B) ZIF-8 at doses below, at and above their determined IC 50 values. Representative normalized alpha parameter for BEAS-2B cells after exposure to (C) MIL-I60 and (D) ZIF-8 at doses below, at and above their determined IC ${ }_{50}$ values. 
respective $\mathrm{IC}_{50}$ value $(60 \mu \mathrm{g} / \mathrm{mL})$ had resistance values similar to the resistance of the control cells over the majority of the exposure time. A slight loss in cell resistance after approximately $36 \mathrm{hrs}$ of exposure was noted for the cells exposed to $20 \mu \mathrm{g} / \mathrm{mL}$ (Figure 3B). Similar to the cells exposed to MIL-160, cells exposed to the high dose of ZIF-8 displayed a complete loss in cellular resistance, thus indicating a loss in the cell monolayer and cellular viability possibly due to changes in cell-substrate interactions. ${ }^{48}$

ECIS analysis also showed that BEAS-2B cells exposed to MIL-160 caused a dose-dependent decrease in the alpha parameter, i.e., cell-substrate interactions. ${ }^{49}$ Specifically, exposure to $800 \mu \mathrm{g} / \mathrm{mL}$ caused an almost complete loss in recording (Figure 3C), while exposure to $200 \mu \mathrm{g} / \mathrm{mL}$ MIL-160 caused values of alpha parameter similar to those of control. Complementary, exposure to $400 \mu \mathrm{g} / \mathrm{mL}$ led to a decrease in the alpha parameter. Cells exposed to 20 and $60 \mu \mathrm{g} / \mathrm{mL}$ ZIF- 8 had similar alpha parameter values as the control cells, while cells exposed to $100 \mu \mathrm{g} / \mathrm{mL}$ displayed a complete loss in alpha and thus a loss in cell-substrate interactions (Figure 3D).

The different degrees of cell-substrate interactions as analyzed by our real-time, high-throughput strategy confirmed the possible different routes of toxicity for the two MOFs, based on their different physicochemical properties. ${ }^{21}$ Specifically, the results suggest that the loss in resistance displayed by BEAS-2B cells exposed to 200 $\mu \mathrm{g} / \mathrm{mL}$ MIL-160 was likely not due to changes in cellsubstrate interactions. In contrast, the similar degrees of decrease in the alpha parameter for cells exposed to 400 and $800 \mu \mathrm{g} / \mathrm{m}$ MIL-160, along with a larger drop in resistance for cells exposed to $200 \mu \mathrm{g} / \mathrm{mL}$, suggest that the loss in resistance is more due to the loss in cell viability or changes in morphology rather than due to cell-substrate alterations. Further, the similarity of the degree of decrease in resistance and alpha parameter for cells exposed to ZIF-8 at all doses suggests that cellsubstrate interactions may be responsible for the cellular toxicity of these particles.

Our assessment is supported by the previous singlepoint studies that identified increased toxicity based on material hydrophobicity, with such characteristic to affect interactions of the specific material with the lipid bilayer ${ }^{40,42}$ and subsequently induce changes in membrane's integrity and cell-responsive signaling. ${ }^{42,43}$ Our results thus demonstrate that the proposed strategy represents an effective means of assessment, with additional identified benefits to classify toxicity based on the times of exposure. Specifically, the early time points associated with changes in cellular behavior could be further used as a means to mitigate additional or concentrated exposures, thus possibly allowing for cellular recovery if exposure stops or is removed.

Our proposed screening assay represents an important step towards detection and assessing MOF-induced deleterious effects in a wider variety of cell lines, with the range of toxic mechanisms to be differentiated based on cell viability, morphology and cell-substrate interactions, respectively. Furthermore, considering that our highthroughput screening strategy indicates that cell death manifests through loss of cell-substrate interactions, our approach could help become an instrumental tool for discovering what specific pathways are actually being targeted, thus complimenting molecular-based hypotheses, however, in a much more reliable and high-throughput fashion. While this current study supports the use of a real-time technique to screen for toxicity, it does not suggest replacement of currently used in vitro toxicity testing strategies provided by the single-point assays but rather seeks to add a valuable primary screening tool that could be supplemented by a variety of other assays to ensure assessment of cellular metabolic activity for instance. In addition, this study was performed using in vitro cell lines; to further understand the toxicological effects of these particles as they relate to human exposure, studies should be done in vivo and on suitable animal models.

\section{Conclusion}

We proposed a screening strategy that allows for differentiation of toxicity in human epithelial cells in a real-time, high-throughput fashion. Our analysis suggests different routes of toxicity to be influenced by MOFs physicochemical characteristics. Our results emphasize the importance of performing toxicological screening assessment of MOFs before their implementation in biomedical applications.

\section{Acknowledgment}

This work was funded by the National Science Foundation (NSF) grant 1454230 and National Institutes of Health (R01-ES022968). Olivia L Rose and Cerasela Zoica Dinu also acknowledge the NSF, DMR1559880.

\section{Disclosure}

The authors report no conflicts of interest in this work. 


\section{References}

1. Arbulu RC, Jiang YB, Peterson EJ, Qin Y. Metal-organic framework (MOF) nanorods, nanotubes, and nanowires. Angew Chem Int Edit. 2018;57:5813-5817. doi:10.1002/anie.201802694

2. Dhakshinamoorthy A, Asiri AM, Garcia H. Catalysis by metalorganic frameworks in water. Chem Commun. 2014;50:1280012814. doi: $10.1039 / \mathrm{C} 4 \mathrm{CC} 04387 \mathrm{~A}$

3. Eddaoudi M. Size almost doesn't matter. Nat Mater. 2007;6:718-719. doi:10.1038/nmat2020

4. Furukawa H, Cordova KE, O'Keeffe M, Yaghi OM. The chemistry and applications of metal-organic frameworks. Science. 2013;341:974-986. doi:10.1126/science.1230444

5. Ma A, Luo Z, Gu C, Li B, Liu J. Cytotoxicity of a metal-organic framework: drug delivery. Inorg Chem Commun. 2017;77:68-71. doi:10.1016/j.inoche.2017.01.004

6. Zhu QL, Xu Q. Metal-organic framework composites. Chem Soc Rev. 2014;43:5468-5512. doi:10.1039/c3cs60472a

7. Kim J, Kim DO, Kim DW, Sagong K. Synthesis of Zn-MOF incorporating titanium-hydrides as active sites binding H-2 molecules. $J$ Solid State Chem. 2015;230:110-117. doi:10.1016/j.jssc.2015.06.034

8. Seoane B, Coronas J, Gascon I, et al. Metal-organic framework based mixed matrix membranes: a solution for highly efficient $\mathrm{CO} 2$ capture? Chem Soc Rev. 2015;44:2421-2454. doi:10.1039/c4cs00437j

9. Li B, Wen HM, Cui YJ, Zhou W, Qian GD, Chen BL. Emerging multifunctional metal-organic framework materials. Adv Mater. 2016;28:8819-8860.

10. Liu Q, Chapman J, Huang AS, et al. User-tailored metal organic frameworks as supports for carbonic anhydrase. Acs Appl Mater Inter. 2018;10:41326-41337. doi:10.1021/acsami.8b14125

11. Salunkhe RR, Kaneti YV, Yamauchi Y. Metal-organic frameworkderived nanoporous metal oxides toward supercapacitor applications: progress and prospects. ACS Nano. 2017;11:5293-5308. doi:10.1021/ acsnano.7b02796

12. Zhang C, Zhang L, Xu GC, et al. Metal organic framework-derived $\mathrm{Co} 3 \mathrm{O} 4$ microcubes and their catalytic applications in $\mathrm{CO}$ oxidation. New J Chem. 2017;41:1631-1636. doi:10.1039/C6NJ02507B

13. Chowdhury MA. Metal-organic-frameworks for biomedical applications in drug delivery, and as MRI contrast agents. $J$ Biomed Mater Res A. 2017;105:1184-1194. doi:10.1002/jbm.a.35995

14. Yang WT, Feng J, Zhang HJ. Facile and rapid fabrication of nanostructured lanthanide coordination polymers as selective luminescent probes in aqueous solution. J Mater Chem. 2012;22:6819-6823. doi: $10.1039 / \mathrm{c} 2 \mathrm{jm} 16344 \mathrm{f}$

15. Taylor KML, Rieter WJ, Lin WB. Manganese-based nanoscale metal-organic frameworks for magnetic resonance imaging. $J \mathrm{Am}$ Chem Soc. 2008;130:14358-+. doi:10.1021/ja803777x

16. Chowdhuri AR, Bhattacharya D, Sahu SK. Magnetic nanoscale metal organic frameworks for potential targeted anticancer drug delivery, imaging and as an MRI contrast agent. Dalton T. 2016;45:29632973. doi: $10.1039 / \mathrm{C} 5 \mathrm{DT} 03736 \mathrm{~K}$

17. Rieter WJ, Taylor KML, An HY, Lin WL, Lin WB. Nanoscale metalorganic frameworks as potential multimodal contrast enhancing agents. $J$ Am Chem Soc. 2006;128:9024-9025. doi:10.1021/ja0627444

18. Beg S, Rahman M, Jain A, et al. Nanoporous metal organic frameworks as hybrid polymer-metal composites for drug delivery and biomedical applications. Drug Discov Today. 2017;22:625-637. doi:10.1016/j.drudis.2016.10.001

19. Filippousi M, Turner S, Leus K, et al. Biocompatible Zr-based nanoscale MOFs coated with modified poly (epsilon-caprolactone) as anticancer drug carriers. Int J Pharmaceut. 2016;509:208-218. doi:10.1016/j.ijpharm.2016.05.048

20. Wang HN, Meng X, Yang GS, et al. Stepwise assembly of metalorganic framework based on a metal-organic polyhedron precursor for drug delivery. Chem Commun. 2011;47:7128-7130. doi:10.1039/ c1cc11932j
21. Tamames-Tabar C, Cunha D, Imbuluzqueta E, et al. Cytotoxicity of nanoscaled metal-organic frameworks. J Mater Chem B. 2014;2:262271. doi:10.1039/C3TB20832J

22. Ruyra A, Yazdi A, Espin J, et al. Synthesis, culture medium stability, and in vitro and in vivo zebrafish embryo toxicity of metal-organic framework nanoparticles. Chem-Eur J. 2015;21:2508-2518. doi:10.1002/chem.201405380

23. Ren F, Yang BC, Cai J, Jiang YD, Xu J, Wang S. Toxic effect of zinc nanoscale metal-organic frameworks on rat pheochromocytoma (PC12) cells in vitro. J Hazard Mater. 2014;271:283-291. doi:10.1016/j.jhazmat.2014.02.026

24. Grall R, Hidalgo T, Delic J, Garcia-Marquez A, Chevillard S, Horcajada P. In vitro biocompatibility of mesoporous metal (III; Fe, $\mathrm{Al}, \mathrm{Cr}$ ) trimesate MOF nanocarriers. J Mater Chem B. 2015;3:82798292. doi:10.1039/C5TB01223F

25. Eldawud R, Wagner A, Dong CB, Rojansakul Y, Dinu CZ. Platform for real-time multi-parametric analysis of cellular behavior postexposure to single-walled carbon nanotubes. Biosens Bioelectron. 2015;71:269-277. doi:10.1016/j.bios.2015.04.044

26. Cadiau A, Lee JS, Borges DD, et al. Design of hydrophilic metal organic framework water adsorbents for heat reallocation. Adv Mater. 2015;27:4775-4780. doi:10.1002/adma.201502418

27. Zhang K, Lively RP, Zhang C, et al. Exploring the framework hydrophobicity and flexibility of ZIF-8: from biofuel recovery to hydrocarbon separations. J Phys Chem Lett. 2013;4:3618-3622. doi:10.1021/jz402019d

28. Park YH, Kim D, Dai J, Zhang Z. Human bronchial epithelial BEAS$2 \mathrm{~B}$ cells, an appropriate in vitro model to study heavy metals induced carcinogenesis. Toxicol Appl Pharm. 2015;287:240-245. doi:10.10 16/j.taap.2015.06.008

29. Borges DD, Normand P, Permiakova A, et al. Gas adsorption and separation by the Al-based metal-organic framework MIL-160. $J$ Phys Chem C. 2017;121:26822-26832. doi:10.1021/acs.jpcc.7b088 56

30. Haydar AL, Abid M, Sunderland HR, Wang S. Metal organic frameworks as a drug delivery system for flurbiprofen. Drug Des Dev Ther. 2017;11:2685-2695. doi:10.2147/DDDT.S145716

31. Zheng HQ, Zhang YN, Liu LF, et al. One-pot synthesis of metal organic frameworks with encapsulated target molecules and their applications for controlled drug delivery. $J$ Am Chem Soc. 2016;138:962-968. doi:10.1021/jacs.5b11720

32. Qian JF, Sun FA, Qin LZ. Hydrothermal synthesis of zeolitic imidazolate framework-67 (ZIF-67) nanocrystals. Mater Lett. 2012;82: 220-223.

33. Pan YC, Liu YY, Zeng GF, Zhao L, Lai ZP. Rapid synthesis of zeolitic imidazolate framework-8 (ZIF-8) nanocrystals in an aqueous system. Chem Commun. 2011;47:2071-2073. doi:10.1039/c0cc05002d

34. Giaever I, Keese CR. Micromotion of mammalian-cells measured electrically. P Natl Acad Sci USA. 1991;88:7896-7900. doi:10.10 73/pnas.88.17.7896

35. Wegener J, Keese CR, Giaever I. Electric cell-substrate impedance sensing (ECIS) as a noninvasive means to monitor the kinetics of cell spreading to artificial surfaces. Exp Cell Res. 2000;259:158-166. doi:10.1006/excr.2000.4919

36. Xiao C, Lachance B, Sunahara G, Luong JHT. An in-depth analysis of electric cell-substrate impedance sensing to study the attachment and spreading mammalian cells. Anal Chem. 2002;74:1333-1339. doi:10.1021/ac011104a

37. Morris W, Stevens CJ, Taylor RE, Dybowski C, Yaghi OM, GarciaGaribay MA. NMR and X-ray study revealing the rigidity of zeolitic imidazolate frameworks. J Phys Chem C. 2012;116:13307-13312. doi: $10.1021 / \mathrm{jp} 303907 \mathrm{p}$

38. Eldawud R, Wagner A, Dong CB, Stueckle TA, Rojanasakul Y, Dinu $\mathrm{CZ}$. Carbon nanotubes physicochemical properties influence the overall cellular behavior and fate. Nanoimpact. 2018;9:72-84. doi:10. 1016/j.impact.2017.10.006 
39. Eldawud R, Reitzig M, Opitz J, et al. Combinatorial approaches to evaluate nanodiamond uptake and induced cellular fate. Nanotechnology. 2016;27. doi:10.1088/0957-4484/27/36/365202

40. Farcal L, Andon FT, Di Cristo L, et al. Comprehensive in vitro toxicity testing of a panel of representative oxide nanomaterials: first steps towards an intelligent testing strategy. PLoS One. 2015;10. doi:10.1371/journal.pone.0127174

41. Vesterkvist PSM, Misiorek JO, Spoof LEM, Toivola DM, Meriluoto JAO. Comparative cellular toxicity of hydrophilic and hydrophobic microcystins on caco-2 cells. Toxins. 2012;4:1008-1023. doi:10.3390/toxins4111008

42. Ibarguren M, Lopez DJ, Escriba PV. The effect of natural and synthetic fatty acids on membrane structure, microdomain organization, cellular functions and human health. Biochim Biophys Acta. 2014;1838:1518-1528. doi:10.1016/j.bbamem.2013.12.021

43. Nor YA, Niu Y, Karmakar S, et al. Shaping nanoparticles with hydrophilic compositions and hydrophobic properties as nanocarriers for antibiotic delivery. ACS Cent Sci. 2015;1:328-334. doi:10.1021/ acscentsci.5b00199

44. Lazaro IA, Lazaro SA, Forgan RS. Enhancing anticancer cytotoxicity through bimodal drug delivery from ultrasmall $\mathrm{Zr}$ MOF nanoparticles. Chem Commun. 2018;54:2792-2795. doi:10.1039/C7CC09739E
45. Wallace WE, Keane MJ, Murray DK, Chisholm WP, Maynard AD, Ong T. Phosopholipid lung surfacant and nanoparticle surface toxicity: lessons from diesel soots and silicate dusts. J Nanopart Res. 2007;9:23-38. doi:10.1007/s11051-006-9159-5

46. Napierska D, Thomassen LCJ, Rabolli V, et al. Size-dependent cytotoxicity of monodisperse silica nanoparticles in human endothelial cells. Small. 2009;5:846-853. doi:10.1002/smll.200800461

47. Osman AF, TF MF, Rakibuddin M, et al. Pre-dispersed organomontmorillonite (organo-MMT) nanofiller: morphology, cytocompatibility and impact on flexibility, toughness and biostability of biomedical ethyl vinyl acetate (EVA) copolymer. Mater Sci Eng C Mater Biol Appl. 2017;74:194-206. doi:10.1016/j.msec.2016.11. 137

48. Xiao CD, Lachance B, Sunahara G, Luong JHT. Assessment of cytotoxicity using electric cell-substrate impedance sensing: Concentration and time response function approach. Anal Chem. 2002;74:5748-5753. doi:10.1021/ac025848f

49. Lo CM, Keese CR, Giaever I. Impedance analysis of MDCK cells measured by electric cell-substrate impedance sensing. Biophys J. 1995;69:2800-2807. doi:10.1016/S0006-3495(95)80153-0
International Journal of Nanomedicine

\section{Publish your work in this journal}

The International Journal of Nanomedicine is an international, peerreviewed journal focusing on the application of nanotechnology in diagnostics, therapeutics, and drug delivery systems throughout the biomedical field. This journal is indexed on PubMed Central, MedLine, CAS, SciSearch ${ }^{\circledR}$, Current Contents ${ }^{\mathbb{R}} /$ Clinical Medicine,
Journal Citation Reports/Science Edition, EMBase, Scopus and the Elsevier Bibliographic databases. The manuscript management system is completely online and includes a very quick and fair peer-review system, which is all easy to use. Visit http://www.dovepress.com/ testimonials.php to read real quotes from published authors. 